\section{Fracturas costales simétricas asociadas a tos}

\author{
DAIANA A. TROVATO ${ }^{1}$, JORGE E. DE SOUSA², \\ JULIO E. BRUETMAN ${ }^{1}$, BÁRBARA C. FINN ${ }^{1}$, PABLO YOUNG ${ }^{1}$
}

\section{Symmetrical rib fractures associated with chronic cough. Report of one case}

Cough may be associated with complications such as syncope, urinary incontinence, pneumothorax, and less frequently, pulmonary hernia and costal fractures. Chronic cough is a cause of rib fractures and when they occur it is likely to affect more than one rib. We report a 53 year-old obese male in treatment with enalapril $10 \mathrm{mg}$ for hypertension with a dry cough lasting five months. He consulted for bilateral chest pain and a Chest $X$ ray examination showed symmetrical fractures in the seventh left and right ribs. Enalapril was discontinued, cough and pain subsided in two weeks.

(Rev Med Chile 2018; 146: 391-393)

Key words: Angiotensin-Converting Enzyme Inhibitors; Chest Pain; Cough; Enalapril; Rib Fractures.
'Servicio de Clínica Médica, Hospital Británico de Buenos Aires, Argentina. ${ }^{2}$ Servicio de Neurología, Hospital Británico de Buenos Aires, Argentina.

Recibido el 31 de octubre de 2017, aceptado el 8 de abril de 2018.

Correspondencia a: Pablo Young Hospital Británico. Perdriel 74 (1280) Buenos Aires, Argentina. Tel 541143096400 Fax 541143043393 pabloyoung2003@yahoo.com.ar

\section{I} a tos es un síntoma común, motivo frecuente de consulta en la práctica ambulatoria. Aunque generalmente es autolimitada, puede estar asociada a complicaciones, sobre todo si es crónica ${ }^{1,2}$. Las complicaciones más conocidas son el síncope, la incontinencia de orina, el neumotórax y, menos frecuentemente, la hernia pulmonar y las fracturas costales ${ }^{3-10}$. La tos puede ocasionar una alteración importante en la calidad de vida. Presentamos un caso de fracturas costales simétricas asociadas a tos por un inhibidor de la enzima convertidora de angiotensina (IECA).

\section{Caso clínico}

Paciente varón de 53 años con antecedentes de hipertensión arterial en tratamiento con enalapril $10 \mathrm{mg}$ /día desde 2009, obesidad mórbida y tos seca de 5 meses de evolución, pero con mayor frecuencia en el último mes, que consultó a nuestro centro en junio de 2015 por dolor punzante a nivel de hemitórax derecho (paraesternal inferior), que aumentaba durante la inspiración profunda y cedía con el reposo. Tres días antes de la consulta agregó dolor de iguales características en hemitórax izquierdo. En el examen físico se constató dolor a la palpación en ambas parrillas costales con predominio de séptimas costillas. Se realizó radiografía de tórax $(\mathrm{Rx} T \mathrm{x})$ de frente y perfil que no mostraba lesiones, y una analítica completa de sangre que fue normal. Debido a la intensidad del dolor ante una Rx Tx normal, se solicitó una tomografía axial computada (TAC) de tórax que confirmó fracturas de los séptimos arcos costales (Figura 1) y mostró ausencia de compromiso óseo metastásico u osteopenia, llegando de este modo a nuestro diagnóstico de fracturas costales simétricas asociadas a la tos. Debido a que el paciente no tenía historia de tabaquismo y descartando goteo postnasal, asma bronquial y reflujo gastroesofágico, se interpretó que la tos crónica fue debida a la utilización de IECA, por lo que se cambió el enalapril por losartán $50 \mathrm{mg} /$ día. Se indicó reposo y un corsé, con el cual el dolor mejoró y desapareció por completo a las dos semanas, estando asintomático al año y medio de aquel cuadro. 


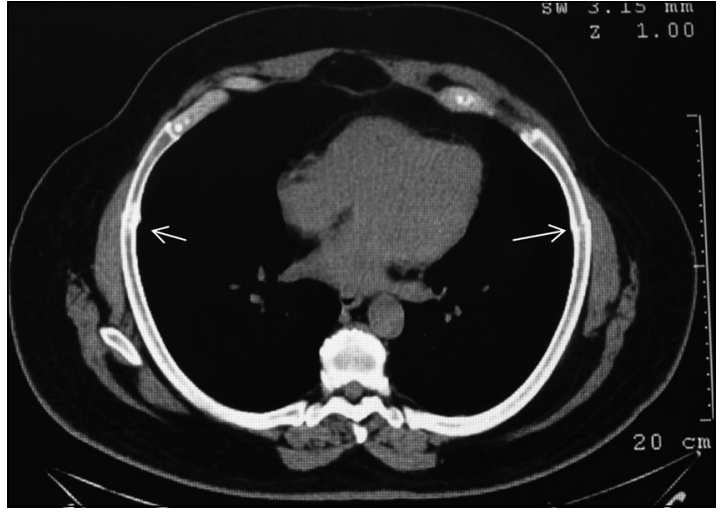

Figura 1. Fracturas simétricas de las séptimas costillas (fechas).

\section{Discusión}

La tos se clasifica en aguda o crónica (TC) de acuerdo al tiempo de duración. Se considera crónica cuando dura más de ocho semanas ${ }^{1,2}$. Se han descripto como causas frecuentes de TC el goteo postnasal, el asma y el reflujo gastroesofágico en pacientes no fumadores o medicados con IECA, ya que estas últimas son causas muy prevalentes de TC, aunque en algunos pacientes se pueden superponer distintas etiologías ${ }^{2}$.

La TC por IECA aparece entre 5 y $20 \%$ de los pacientes que reciben la medicación ${ }^{1,11}$. Este efecto no es dosis dependiente y puede aparecer a los pocos días de iniciado el tratamiento, como así también luego de varios meses. El mecanismo implicado es la elevación de la bradiquinina que es metabolizada por la enzima convertidora. La tos desaparece dentro de las cuatro semanas de suspendido el fármaco ${ }^{1}$. Hasta el momento no se han reportado fracturas costales por tos debidas a IECA.

Se han comunicado como causa de fracturas costales el trauma torácico y algunos deportes como remo, béisbol, alpinismo y golf ${ }^{3,6}$. La TC es una causa en creciente reconocimiento de fracturas costales y, cuando ocurre, es probable que afecte a más de una costilla (múltiples). Las fracturas costales producidas por la tos pueden ocurrir en sujetos por lo demás normales, aunque su desarrollo debería, por lo menos, suscitar la sospecha de que la fractura sea patológica, tal y como ocurre en el mieloma múltiple, la osteoporosis y las metástasis osteolíticas ${ }^{5}$.
El primero en describir la tos como causa de fracturas costales fue Robert J. Graves, en $1843^{12}$. Posteriormente, Webb y cols., en 1923, describieron un caso de fractura costal secundaria a tos ${ }^{13}$. Hasta la fecha se han publicado en la literatura mundial aproximadamente doscientos cincuenta casos $^{1-16}$.

Hanak y col., en el año 2005, describieron una serie retrospectiva de 54 pacientes con fracturas costales inducidas por tos durante un período de 9 años $^{14}$. Más de $75 \%$ de los pacientes fueron mujeres, la edad media al diagnóstico fue 55 años. La mayoría de los pacientes (85\%) tenía TC. Los 54 pacientes comunicados presentaban 112 fracturas, 50\% (27 pacientes) de ellos tuvo una sola fractura, y en otro $50 \%$ se identificó dos o más fracturas. De estos últimos, 14 pacientes tuvieron dos fracturas, 6 pacientes tuvieron 3 fracturas, 5 pacientes tuvieron 4 fracturas, 2 pacientes tuvieron 5 o más fracturas costales. Solo 14 pacientes tuvieron fracturas bilaterales. A lo largo de la costilla, las fracturas se situaron a nivel lateral en $50 \%$ de los pacientes, con aspecto de callo óseo anterior en $26 \%$ y posterior en $24 \%$. La sexta costilla fue la más afectada de ambos lados, seguida por la 5ta, 7ma y 9na. Ninguno de los pacientes tenía cáncer como enfermedad asociada ${ }^{14}$.

Sano y col. describieron recientemente 14 pacientes con fracturas costales por tos ${ }^{15}$. La edad media fue de 39,5 años, diez eran mujeres y hubo una sola fractura en 9 pacientes y 5 tuvieron fracturas múltiples. La costilla más comúnmente afectada fue la décima y los pacientes no tuvieron enfermedades subyacentes.

La Rx Tx no detecta hasta $60 \%$ de las fracturas, ya que estas son visibles más tardíamente, cuando ya existe formación del callo óseo ${ }^{1,3,16}$. En cambio, el centellograma y la TAC de tórax son muy sensibles ${ }^{16}$. El centellograma no es específico, ya que hallazgos similares se observan en fracturas antiguas, tumores óseos primarios, como el osteocondroma, y enfermedad metastásica. En la TAC se observa ocasionalmente en las fracturas recientes la disrupción de la corteza costal. También existe un área sutil de esclerosis ósea $\mathrm{u}$ osteólisis $\mathrm{y}$, eventualmente, la presencia de un hematoma subpleural y un pequeño fragmento óseo desplazado. Cuando la fractura se ha consolidado, se observa la formación del callo óseo, que consiste en un puente perióstico externo y un callo intramedular. Finalmente, el remodelado 
luego de la fractura puede dejar una deformidad permanente. La TAC puede poner de manifiesto enfermedades subyacentes, como tumores óseos primarios o metastáticos.

El tratamiento suele ser conservador con antiinflamatorios, antitusivos y eventualmente un corsé para inmovilización $n^{1,3,7,15}$.

Este cuadro debe considerarse entre los diagnósticos diferenciales de dolor torácico en un paciente con tos, aun con radiografía de tórax o de parrilla costal normales.

\section{Referencias}

1. Achilleos A. Evidence-based Evaluation and Management of Chronic Cough. Med Clin North Am 2016; 100: 1033-45. (Review).

2. Irwin RS, Madison JM. The diagnosis and treatment of cough. N Engl J Med 2000; 343: 1715-20.

3. Bosio M, Young P, Finn BC, Spina JC, Quadrelli SA, Chertcoff FJ. Fracturas costales múltiples asociadas a tos. Medicina (B Aires) 2008; 68: 380-2.

4. De Maeseneer M, De Mey J, Debaere C, Meysman M, Osteaux M. Rib fractures induced by coughing: an inusual cause of acute chest pain. Am J Emerg Med 2000; 18: 194-7.

5. Vrbanić TS, Novak S, Sestan B, Tudor A, Gulan G. A case of pathological rib fractures: focal osteolysis or osteoporosis? West Indian Med J 2008; 57: 178-81.

6. Karlson KA. Rib stress fractures in elite rowers: a case series and proposed mechanism. Am J Sports Med 1988; 26: 516-9.

7. Kawahara H, Baba H, Wada M, Azuchi M, Ando M, Imura S. Multiple rib fracture associated with severe coughing-a case report. Int Orthop 1997; 21: 279-81. (Review).

8. Connolly LP, Connolly SA. Rib stress fractures. Clin Nucl Med 2004; 29: 614-6.

9. Jamard B, Constantin A, Cantagrel A, Mazières B, Laroche $\mathrm{M}$. Multiple rib fractures caused by coughing in a young woman without bone loss. Rev Rhum Engl Ed 1999; 66: 237-8.

10. Begley A, Wilson DS, Shaw J. Cough fracture in the first rib. Injury 1995; 26: 565-6.

11. Hallberg P, Nagy J, Karawajczyk M, Nordang L, Islander G, Norling P, et al. Comparison of Clinical Factors Between Patients With Angiotensin-Converting Enzyme Inhibitor-Induced Angioedema and Cough. Ann Pharmacother 2016; 25: 1-8.

12. Young P, Finn BC, Bruetman JE. La enfermedad de Graves, signos y síntomas. An Med Interna 2007; 24: 505-8.

13. Webb GB, Gilbert BG. Ribs fractured by coughing. JAMA 1923; 81: 25-9.

14. Hanak V, Hartman TE, Ryu JH. Cough-induced rib fractures. Mayo Clin Proc 2005; 80: 879-82.

15. Sano A, Tashiro K, Fukuda T. Cough-induced rib fractures. Asian Cardiovasc Thorac Ann 2015; 23: 958-60.

16. De Maeseneer M, De Mey J, Lenchik L, Everaert H, Osteaux M. Helical CT of rib lesions: a pattern-based approach. Am J Roentgenol 2004; 182: 173-9. (Review). 